Musées, Patrimoine et Culture scientifiques et techniques

$195 \mid 2021$

mai-juin 2021

\title{
Le musée exploré. L'écriture de terrain au service de la découverte culturelle
}

\section{Hervé Moëlo}

\section{OpenEdition \\ Journals}

Édition électronique

URL : https://journals.openedition.org/ocim/4325

DOI : $10.4000 /$ ocim.4325

ISSN : 2108-646X

Éditeur

OCIM

Édition imprimée

Date de publication : 1 mai 2021

Pagination : 16-21

ISSN : 0994-1908

Référence électronique

Hervé Moëlo, «Le musée exploré. L'écriture de terrain au service de la découverte culturelle », La Lettre de l'OCIM [En ligne], 195 | 2021, mis en ligne le 01 mai 2021, consulté le 13 juin 2022. URL : http:// journals.openedition.org/ocim/4325; DOI : https://doi.org/10.4000/ocim.4325

Ce document a été généré automatiquement le 13 juin 2022.

Tous droits réservés 


\section{Le musée exploré. L'écriture de terrain au service de la découverte culturelle}

Hervé Moëlo

Dessins de jeunes visiteurs du Muséum de Nantes (montage).
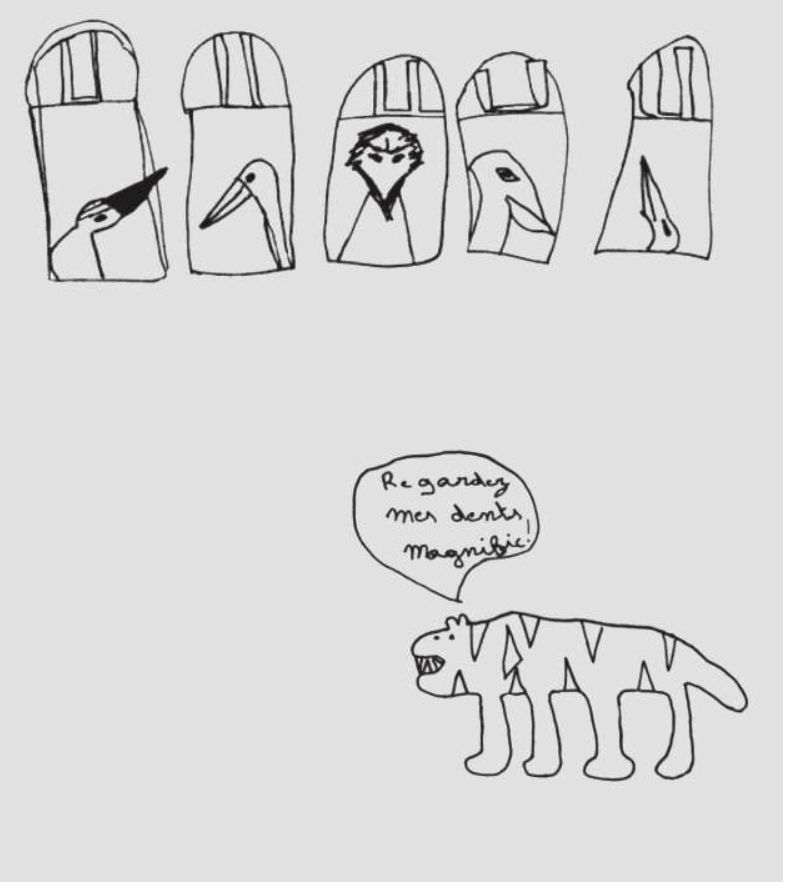

(c) DR

1 «Ce que tu ne connais pas par toi-même, tu ne le connais pas». 

un ancien chantier naval ? Un jardin et un musée?

4 Ce sont quelques-uns des lieux où nous menons des projets d'écriture de terrain avec des enfants. Cette démarche du Centre de ressources Ville de Nantes ${ }^{1}$ a l'avantage d'être transportable partout. Écriture nomade, écriture de plein air, écriture portative, écriture tout terrain... Les termes ne manquent pas, les lieux à explorer non plus. Elle crée même une forme de vertige : tous les lieux sont donc explorables? Nous n'en aurons donc jamais fini?

Depuis une quinzaine d'années, nous emmenons des classes de CE2, CM1, CM2 partout où cela est possible ${ }^{2}$. Pour tous ces lieux si différents, la démarche est la même : entraîner les enfants dans un projet de découverte par l'exploration, rendu plus intense par un travail d'observation et de prise de note, écrite et dessinée. Par la suite, d'autres temps d'écriture en classe permettent de «surfer » sur l'énergie de ces explorations qui ont le mérite de produire de l'enthousiasme et une forte envie d'écrire et de dessiner.

La philosophie de l'écriture de terrain renvoie aux propos de Bertold Brecht: pour connaître un lieu par soi-même, il faut fouiller, chercher, trouver ce qui nous intéresse.

\section{Le Muséum de Nantes, laboratoire de projets d'exploration}

7 Le muséum d'Histoire naturelle a permis d'expérimenter différentes manières de faire ${ }^{3}$. Il a fallu quelques années et plusieurs classes pour que le projet Le musée exploré se règle, s'organise, se précise et arrive à maturité. Simple les premières fois, il a fini par s'enrichir au fil des années, en nous révélant quatre points essentiels. 
Couverture du recueil de textes écrits par les élèves de CM1 de l'école publique Sully à Nantes, dans le cadre de la classe lecture écriture organisée par le Centre ressources Ville de Nantes et avec le concours de l'Inspection académique de Loire-Atlantique et du muséum de Nantes (1999).

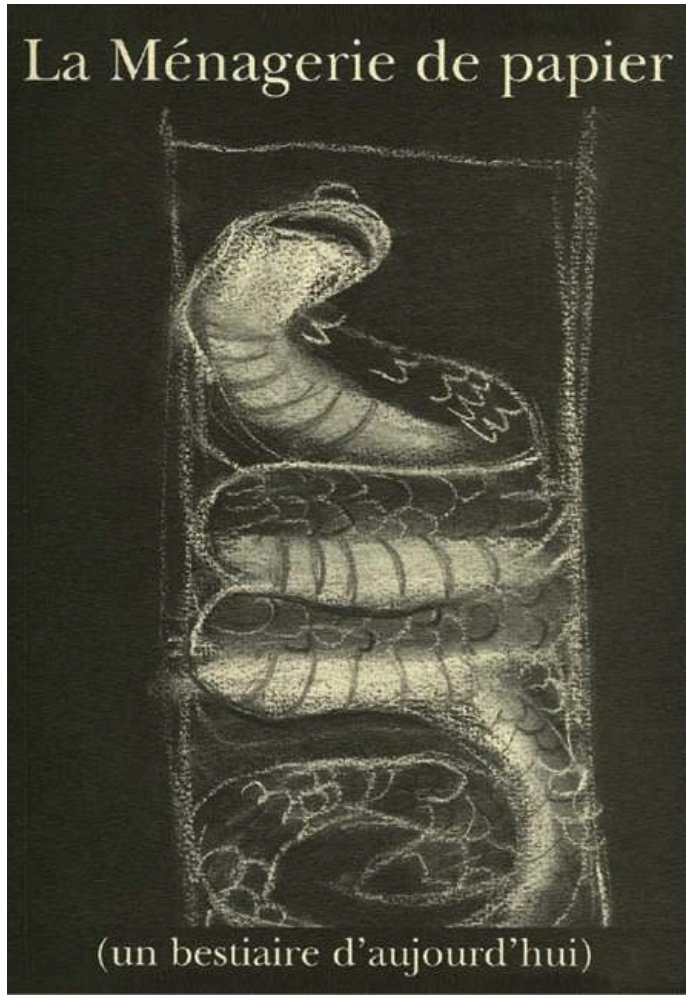

(C) Ville de Nantes

\section{Le choix de l'observation}

Il s'agit avant tout de laisser à chaque enfant le choix de ce qu'il va observer, sans l'imposer a priori. Ce principe est fondamental dans tous les projets d'écriture de terrain. Si on laisse du temps pour parcourir une salle de musée et trouver la pièce de l'exposition qui va "me parler », on a l'assurance que les développements qui suivront seront productifs car porteurs de sens pour chacun. Le choix se fait toujours pour de bonnes raisons. Il fait écho à des raisons personnelles qui vont alimenter les émotions, les pensées, les rêveries et les envies de création graphique, écriture et dessin.

"J'ai choisi ce fossile parce qu'il est tout petit et mystérieux, écrit Marvin. Il ressemble à une puce électronique. Je crois qu'il vivait sur terre et je crois qu'il passait inaperçu de ses proies». La projection dans l'objet commence dès cet instant.

Ce principe du choix ne peut se faire qu'à une seule condition: délimiter l'espace à explorer pour de petits groupes. Bref, le musée doit accepter cette contrainte: ne pas tout montrer aux enfants. Nos explorations permettent de s'installer dans le lieu et de faire qu'on s'y sente bien, plutôt que de le parcourir d'un bout à l'autre. Pas de visites au pas de charge, mais du temps pour s'arrêter, s'installer confortablement, physiquement et psychiquement, dans l'espace et dans le temps. 


\section{L'exploration des coulisses}

11 Elle est devenue une activité fondamentale. Si je viens dans le musée en tant que visiteur lambda, je découvre ce que tout le monde découvre. Si je viens en explorateur de terrain, je vais pouvoir ouvrir ces portes «interdites au public»! Vous la sentez, la différence de statut? Le musée s'ouvre à moi !

Je vous rassure. Il ne s'agit pas de laisser des "hordes» d'enfants envahir tous les espaces sans aucun contrôle: tout cela est organisé avec une grande précision. Au muséum, certains explorent la salle de pause, d'autres les vestiaires des agents d'accueil, situés sous la salle des vivariums, ou les bureaux, dont celui du directeur, ou la bibliothèque ou le laboratoire des collections et de taxidermie. À chaque fois, les habitants des lieux expliquent leur travail et répondent aux questions. Un temps d'exploration, de dessin et d'écriture permettent de scruter le lieu.

Dans les vestiaires, Mariem note: "Petite pièce, petite douche avec casier, table à manger, frigo, micro-onde, café, placard, lave à beau (sic)». Dans la bibliothèque scientifique, Fafanny écrit : «ici, il y a une odeur ancienne de livres anciens. J'ai vu des livres très anciens. En haut il y avait des gens qui couraient. Ici il n'y a pas de bruit."

Notons que le temps d'observation doit être dissocié du temps d'explication car ils sont très différents. Il faut toujours négocier, avec autant de diplomatie que possible, pour obtenir de nos hôtes un temps sans commentaire ni explication, pour permettre de voir ces détails et sentir ces atmosphères qu'on ne peut pas capter quand quelqu'un parle et guide nos perceptions.

\section{L'exploration des extérieurs}

15 C'est une autre activité importante. Tout musée est un bâtiment inscrit dans une globalité urbaine et historique. La première chose que l'on découvre avant d'y entrer, ce sont les rues qui nous y mènent, les bâtiments et les espaces qui l'entourent. Paradoxalement, pour bien connaître un musée, il faut donc ne pas y entrer! Je veux dire, il faut savoir en sortir pour le regarder du dehors, noter-dessiner des détails, sa façade, son environnement. Il est également intéressant de noter le nom des rues qui l'entourent, d'observer les immeubles, les fenêtres, les façades qui sont dans son voisinage. Ce sont des éléments riches, $\mathrm{y}$ compris pour l'imaginaire à venir.

16 Un temps est prévu pour marcher autour du muséum. Issam note que "cette façade est très belle et très sculptée. Il y a des décorations, des dessins différents. Il y a des petits enfants, des femmes, des hommes. On voit que les fenêtres sont petites. Il y a des tigres avec des ailes de chaque côté». 
Bestiaire illustré par les éléves des écoles Chêne d'Aron, Henri Bergson et Jean Zay dans le cadre d'un projet animé par le Centre ressources Ville de Nantes, le Muséum de Nantes, la Compagnie Paq'la Lune et M. Thuillier auteur-plasticienne (2013).

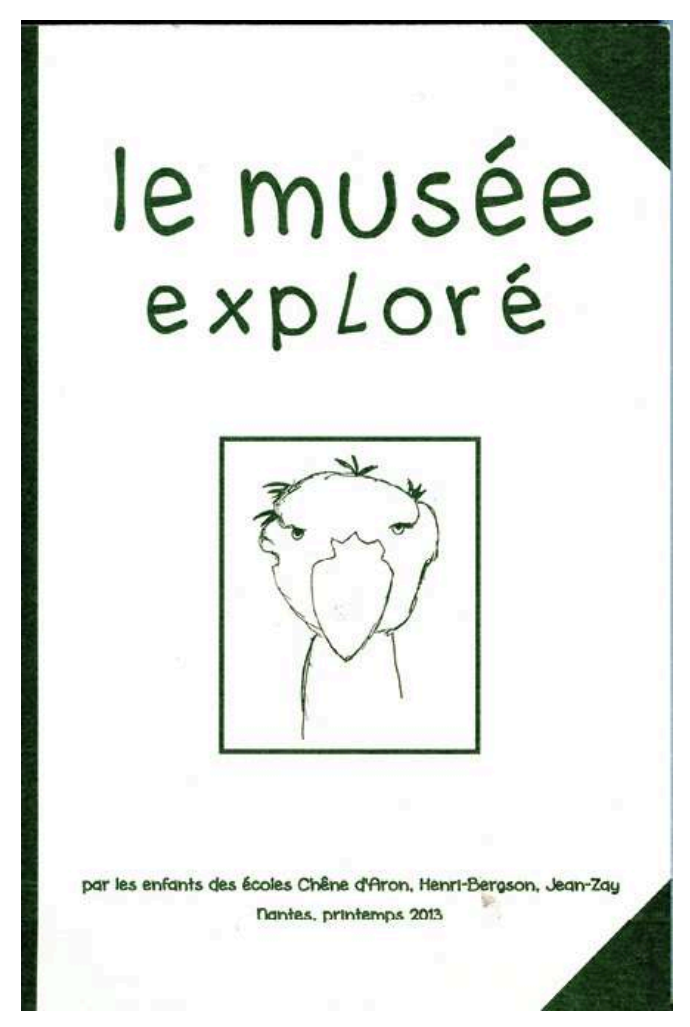

(c) Ville de Nantes

Dessins et textes d'enfants à propos de la façade du Muséum de Nantes.

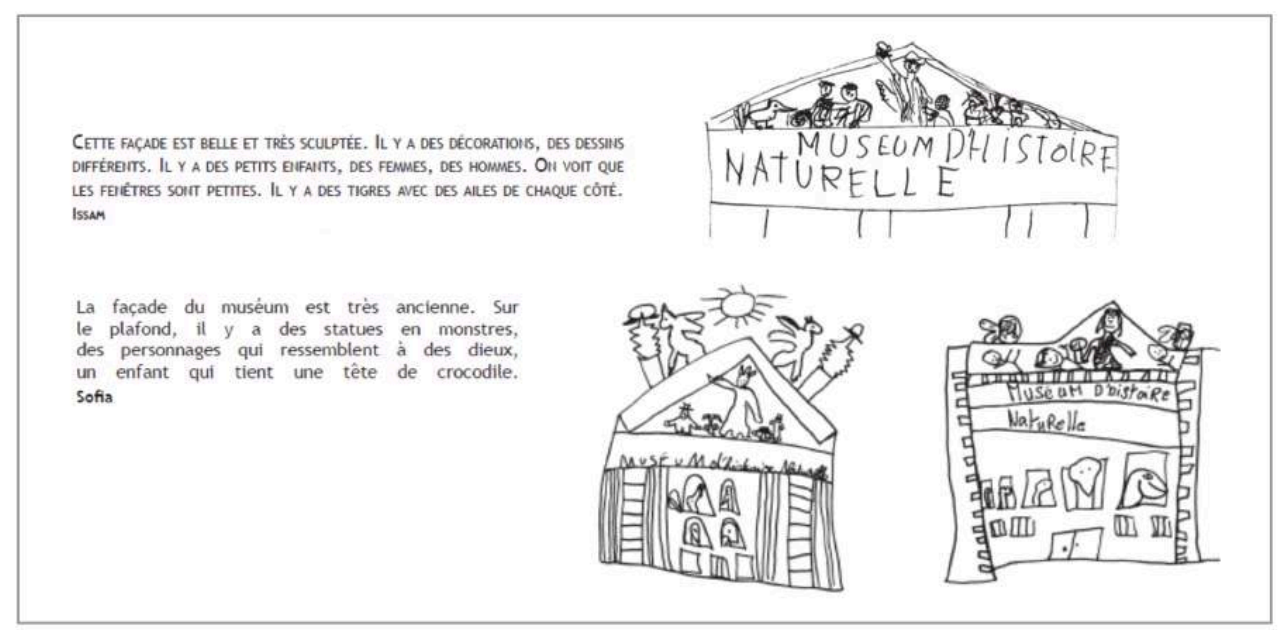

(c) Ville de Nantes

\section{Les rencontres}

Elles donnent enfin toute leur cohérence à la découverte d'un musée. Ce lieu n'est-il pas avant tout un lieu où travaillent toutes les personnes qui le font vivre? Le Muséum de 
Nantes recouvre une dizaine de métiers pour une quarantaine d'agents. Il faut alors faire des choix pour se concentrer sur trois ou quatre professions. permet de donner la parole aux personnes, aux outils, aux lieux, aux bâtiments. Là encore, c'est chaque enfant qui va choisir ce qui l'a le plus marqué, ce qui lui permettra d'avoir plus de choses à écrire sur le sujet.

À partir des prises de notes et des dessins, chacun va choisir ce qu'il veut développer selon le concept du "don de l'imagination exacte» de l'historien Albrecht Betz. On va imaginer certes, mais dans le cadre contraint de la réalité observée : l'animal observé, que pense-t-il ? Comment vit-il sa vie ici? A-t-il des relations privilégiées avec son voisinage ? Avec le personnel du muséum ? Et la nuit, que se passe-t-il ? Une histoire est inventée par une classe : tous les oiseaux partent pour une fête nocturne dans la petite Amazonie, marais urbain, près de leur école du quartier de Malakoff. Ils reviennent le matin. Épuisés, mais heureux.

D'autres animaux n'ont pas cette chance: «Dans le musée, je m'ennuie. J'en ai marre de regarder les gens qui passent. (...) Quel dommage, on ne peut pas respirer! On est toujours dans la même position. On a des courbatures!» (Mathéo). Ou bien encore : «Ma vie au musée, c'est triste! Tout le monde m'admire, matin midi et soir et je n'ai même pas le temps de fermer un ceil. Je ne peux pas respirer. Je suis enfermé dans une vitrine. Arggg! (...) C'est le désespoir total " (Dalile).

24 Au cours de ces projets, les enfants écrivent beaucoup. La quantité est au rendez-vous, souvent au-delà de nos attentes et des volumes habituels de productions en classe. Évidemment, plus on écrit, plus on risque de faire des fautes mais l'objectif du projet ne porte ni sur l'orthographe, ni sur la syntaxe. Plutôt que de répondre à une consigne stricte, il s'agit ici de donner envie d'écrire et de dessiner, en trouvant les idées et l'inspiration autour de soi (observation) et en soi (imagination exacte). Le temps du projet, nous mettons entre parenthèse les difficultés linguistiques, sans pour autant les ignorer. Plus les enfants écrivent, mieux c'est. Chaque enseignant peut, en parallèle, reprendre une des productions écrites pour la retravailler en classe. 
$\mathrm{Au}$ terme du projet, après quatre ou cinq mois, une sélection des textes des classes seront diffusés par une publication ${ }^{4}$ et par une mise en voix travaillée avec des comédiens de la compagnie nantaise PaQ'la Lune. C'est l'occasion de réunir tous les participants au projet.

Une véritable chorale de lecture est mise en place qui proclame des textes écrits par tous et par chacun. Chaque classe devient un auteur collectif. Ce phénomène d'un groupe d'enfants qui parvient à parler d'une même voix a un effet très puissant.

Guide réalisé à l'attention des visiteurs, intégrant le plan du musée, les spécimens à ne pas manquer et des informations scientifiques.

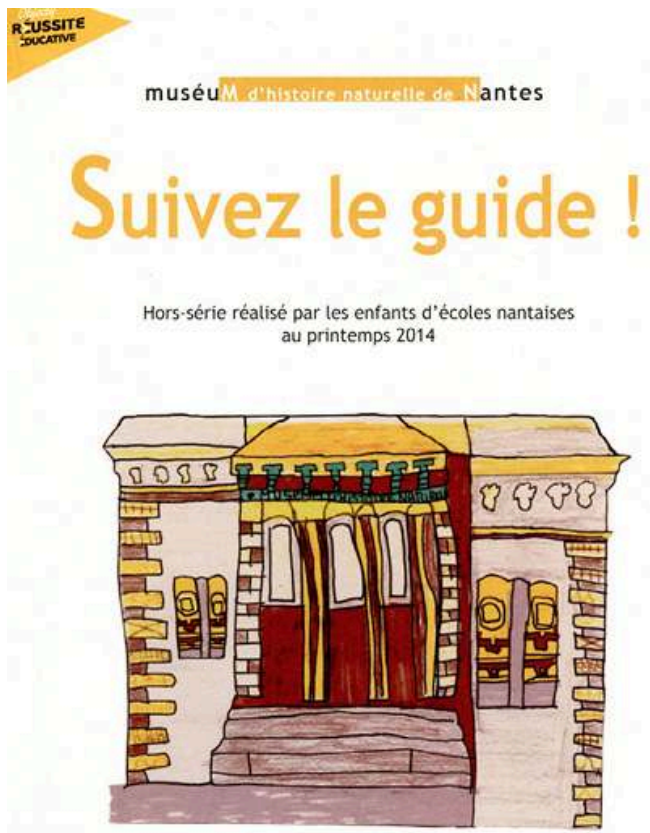

\section{1 lantes}

(c) Service des publics du muséum de Nantes

\section{Le musée pris dans sa globalité et sa matérialité}

La démarche considère le musée dans sa globalité, comme un lieu social où des hommes et des femmes vivent et travaillent, comme un élément urbain inséré dans un quartier. Nous décentrons le cœur de la démarche habituelle de la visite du musée en considérant que les collections présentées ne sont pas l'élément unique de la démarche culturelle.

Il est tout aussi important d'appréhender ce lieu dans sa matérialité, de ne pas le considérer seulement comme un lieu d'exposition mais aussi comme un bâtiment avec des recoins, des sous-sols, des toilettes, des mystères, des couloirs... Comment fonctionne-t-il ? Qui sont ceux qui s'en occupent? Qu'ont-ils à en dire ? Quels sont leurs horaires, leurs contraintes, leurs plaisirs, leurs difficultés ? C'est une autre manière de « comprendre » le lieu, en complément avec ce qu'on y montre. 
E

Cette matérialité est un atout. Pour les enfants de ces projets, dont la majorité ne sont pas familiers des musées, c'est une approche précise et concrète qui leur permet de ne pas être perdus dans des abstractions culturelles.

Plusieurs classes ont été invitées à écrire un guide du muséum: Suivez le guide !5. Extrêmement subjectif et imaginatif, il a été distribué aux visiteurs pour les encourager à venir y vivre la même expérience. Les slogans fusent : «Dans la salle de zoologie, vous ne tomberez pas dans l'ennui !», "Ça brille, c'est grand, c'est différent, on vous y attend!», «Petits curieux, venez nombreux, vous en prendrez plein les yeux!».

\section{Et du côté du muséum ?}

Au-delà d'un bon souvenir de médiation, ce projet a réuni trois ingrédients essentiels de notre action culturelle : laisser la parole aux enfants, leur proposer de vraies missions, et plus largement, faire en sorte que les visiteurs se sentent un peu chez eux au muséum.

Ce projet a naturellement mobilisé l'équipe des médiateurs mais aussi associé tous les collègues qui ont voulu prendre part à l'organisation des journées et se prêter au jeu des observations des élèves. Au fil de leur journée d'exploration, les enfants nous ont côtoyés, observés, questionnés... Il était important de leur ouvrir les portes, de les emmener dans nos bureaux, qu'ils découvrent nos lieux de vie, qu'ils se perdent dans le labyrinthe des couloirs.

Fierté d'être non plus des visiteurs comme les autres mais des invités accueillis dans notre grande maison. Ce lieu que nous habitons tous les jours, un lieu de travail certes, est un espace de découverte, « magique » nous disent certains visiteurs. Nous, les professionnels, avons parfois tendance à l'oublier.

Après leur exploration au muséum, les élèves ont continué à écrire et à dessiner en classe, accompagnés par l'équipe du CRV et avec une demande précise du muséum : produire un guide pour les visiteurs. Un vrai guide avec le plan du musée, les spécimens à ne pas manquer, des informations scientifiques, un programme des actions culturelles. Tiré à 5000 exemplaires, Suivez le guide! ressemble beaucoup à notre plaquette institutionnelle. C'est du sérieux!

Chaque élève en reçoit un exemplaire. Fierté d'avoir répondu à une commande du muséum.

Ce guide a ensuite été distribué à l'accueil du musée. Des visiteurs ont donc suivi les conseils avisés des enfants. Il a aussi inspiré des enseignants pour développer des activités alliant science et langage au muséum.

Dans notre jargon professionnel, ne serait-ce pas là une action de co-construction réussie?

Merci aux élèves, aux collègues du CRV et du muséum.

\section{Sylvie Le Berre}

Service des publics du Muséum de Nantes 
Responsable du Service des publics - Directrice adjointe

\section{Transformer la « visite » en « présence »}

31 L'écriture de terrain, considère a priori les espaces explorés, comme des lieux d'enrichissement et de dialogue. Voilà qui mérite d'être mis encore plus en avant en ces périodes de confinement et de distanciation sociale. Il est fort probable que la double question de la redécouverte et de la reconquête des espaces publics va devenir urgente. Il s'agit de développer une attention plus grande aux espaces sociaux. En nous immergeant plusieurs heures dans un musée avec des enfants, mais aussi dans un jardin, dans une bibliothèque, dans une rue, nous apprenons à voir, à regarder, à mieux comprendre et sentir ce qui s'y passe. Nous apprenons aussi à prendre contact avec les personnes qui passent ou qui travaillent là. La découverte de leurs vies, de leurs conditions de travail est une chose précieuse.

Tout ce temps passé produit un sentiment de familiarité avec le lieu. Nous travaillons la question de la présence, à une époque où l'absence semble se développer au grand galop : numérisation et dématérialisation généralisées, travail à distance, existence en visioconférence, écart grandissant entre la prescription de l'encadrement et la réalité du travail, omniprésence du téléphone...

Nous avons la sensation d'avoir été présents « ici », d'avoir vraiment fait connaissance avec l'endroit, de s'y être senti un peu chez soi. Ne serait-ce pas là une des définitions du mot «habiter»? Plutôt que d'être de passage, nous avons demeuré, faisant connaissance avec cette salle, ces animaux, développant aussi une complicité avec les agents d'accueil du muséum. C'est une manière de transformer la "visite » en " présence ». Alors, elle apparaît, cette sensation de connaître ce lieu par soi-même. Le musée devient un lieu vivant, familier et attachant. On y pense. On l'écoute. Il nous parle. On a envie d'y revenir.

\section{NOTES}

1. Le Centre de ressources Ville (CRV) est un espace de développement, de coordination, d'animation de projets et de formation de la Direction de l'Éducation de la Ville de Nantes. Son domaine : la lecture-écriture et l'éducation artistique et culturelle (EAC).

2. D'autres projets que celui relaté dans cet article sont nés de cette expérience de terrain, en particulier sur le thème du travail : quel rapport des enfants entretiennent-ils avec le travail ? Comment voient-ils les anciens chantiers navals de Nantes ? Comment vivent-ils la rencontre avec les anciens ouvriers?

Découvrez l'article : Attention travail ! L'écriture de terrain ou l'utopie de la présence : https://projetseducation.nantes.fr/documents

3. Réflexion et projets menés avec Sylvie Le Berre, Directrice adjointe du Muséum et son équipe. 
4. Avec des tirages variables, entre 500 et 2000 exemplaires, la collection continue à être diffusée gratuitement, notamment dans les formations, auprès de ceux qui s'intéressent de près aux projets d'écriture.

5. Pour un envoi gratuit des livrets Suivez le guide ! et Le Musée exploré : herve.moelo@mairienantes.fr

\section{RÉSUMÉS}

Observer-analyser-écrire permet de porter l'attention et s'installer dans le présent pour imaginer-interpréter-comprendre le lieu, les personnes y travaillant ou visitant et les objets. Le responsable du Centre de ressources Ville de Nantes dévoile les principes et les réalités de cette approche sensible et partagée, à partir du projet mené avec le Muséum de Nantes.

\section{INDEX}

Mots-clés : politique culturelle, pratique culturelle, éducation populaire, organisation d'éducation populaire

\section{AUTEUR}

\section{HERVÉ MOËLO}

Responsable du Centre de ressources Ville (CRV) à la Direction de l'Éducation de la Ville de Nantes.

herve.moelo@mairie-nantes.fr 